\title{
A Higher Control Center of Locomotor Behavior in the Drosophila Brain
}

\author{
Roland Strauss ${ }^{a}$ and Martin Heisenberg \\ Theodor-Boveri-Institut für Biowissenschaften, Lehrstuhl für Genetik, Am Hubland, W-8700 Würzburg, Germany
}

In order to elucidate the behavioral significance of the central complex (CC), we have examined walking in 15 Drosophila mutant strains belonging to eight independent $X$-linked genes that affect the structure of the CC. Compared to four different wild-type strains, all are impaired either in a general or in a paradigm-dependent manner. Behavioral deficits concern walking activity, walking speed, or "straightness of walking" as measured in an object fixation task, in fast phototaxis, and in negative geotaxis.

Behavioral deficits of three strains with mutations in different genes were studied in detail using mosaic analysis and high-speed cinematography. In all cases the focus for declining walking activity is located in the brain and is fully correlated with the respective defect of the CC. A high correlation between the degree of the behavioral impairment and the severity of the structural defect in two strains further adds to the evidence. Declining walking activity is not an unspecific side effect of structural brain defects, as steady walking is observed in structural mutants of the visual system and mushroom bodies.

In mutant tlies no-bridge ${ }^{\mathrm{Ks} 49}$ (nob), step size as a function of the stepping period is reduced. The focus of the resulting reduced average and maximum walking speeds resides in the brain and, again, the behavioral impairment fully correlates with the structural defects of the CC.

While no indication is found for a role of the $C C$ in setting up the basic stepping rhythm in straight walking (a respective phenotype in mutant central-complex ${ }^{\mathrm{k} 181}$ flies resides in the ventral ganglion), a role in turning and start/stop maneuvers is suggested by aberrations in the stepping pattern of nob flies during such episodes.

IKey words: Drosophila melangoster, genetic dissection, central complex, structural brain mutants, walking behavior, mosaic analysis]

What makes behavior so interesting is not so much what the behaving organism actually does but why, when, and how it does it. It is for this that higher centers of the nervous system are needed. Vertebrate spinal cord and the thoracic and abdominal ganglia of arthropods harbor many motor programs such

\footnotetext{
Received July 29, 1992; revised Oct. 28, 1992; accepted Nov. 2, 1992.

This work is dedicated to Franz Huber, who pioneered central complex research, in honor of his 65 th birthday. We thank $M$. Dickinson for valuable comments on the manuscript, C. S. Goodman for kind advice, and G. Neckermann, T. Wanke, and $U$. Bolze for excellent stock maintenance and skillful technical assistance. This study was supported by DFG Grant He 986 (M.H.).

Correspondence should be addressed to $M$. Heisenberg at the above address.

${ }^{a}$ Present address: Max-Planck-Institut für biologische Kybernetik, Spemannstrasse 38, W-7400 Tübingen, Germany.
}

Copyright @ 1993 Society for Neuroscience $0270-6474 / 93 / 131852-10 \$ 05.00 / 0$ as righting, grooming, or walking, yet a spinal cat and a decapitated cockroach are of limited interest to an ethologist. In insects, in terms of systematic behavioral analysis, much is known about the influence of sensory organs on behavior, but little about what distinguishes the brain from the lower centers of the CNS. Disregarding the optic and antennal lobes, two outstanding structures in the insect brain are unique with respect to other ganglia: the mushroom bodies and the central complex $(\mathrm{CC})$. Experiments on bees (Menzel et al., 1974) and flies (Heisenberg et al., 1985) indicate that the mushroom bodies function as associative centers. For the $\mathrm{CC}$, anecdotal data from surgery (e.g., Huber, 1960) and electrical stimulation (Otto, 1971) havc suggested a role in the regulation of behavioral activity (for a review, see Homberg, 1987). Here we report new evidence from genetic dissection in Drosophila confirming this general idea and more specifically and systematically showing that the $\mathrm{CC}$ controls certain features of locomotor behavior.

\section{Materials and Methods}

Flies. Mutant strains had been isolated by mass histology (Heisenberg and Böhl, 1979) following ethylmethane sulfonate mutagenesis of wildtype Berlin [WT(B)] flies. As the only exception, agnostic $\mathrm{C}^{\mathrm{X}}\left(\operatorname{agn}^{\mathrm{x}}\right)$ has been isolated from a wild population (E. V. Savvateeva, personal communication to M.H.). Mutant behavior was compared to wild-type strains WT(B), Canton-S (C-S), Oregon-R (O-R), and Lausanne-S (L-S). nobridge $^{\mathrm{KS49}}\left(\mathrm{nob}^{\mathrm{KS49}}\right)$, central-body-defect ${ }^{2254}\left(\mathrm{cbd}^{2254}\right)$, central-brainderanged ${ }^{892}\left(c^{8} b^{892}\right)$, and ellipsoid-body-open (ebo $\left.{ }^{1041}\right)$ males were cultured with $C(1) D X, y w f / Y$ females. All strains were raised on standard Drosophila medium (cornmeal, agar, molasses, yeast, nipagin) under constant light-dark cycles (light from 7 A.M. to 7 P.M.) at $23^{\circ} \mathrm{C}$ and $80 \%$ relative humidity.

In order to obtain mosaic flies, $y w^{\mathrm{a}} n o b^{\mathrm{KS} 49} f$. $n o b^{\mathrm{Ks} 49} s n^{3}, s n^{3} c b d^{2254}$ or central-complex ${ }^{\mathrm{KS} 181} \mathrm{f}\left(\mathrm{cex}^{\mathrm{KS} 181} f\right)$, respectively, were mated to $R(1)$, wr $/ y w$ females and the offspring was screened for individuals with appropriate distribution of external markers (Hinton, 1955).

In "Buridan's paradigm" (Götz, 1980), flies walk spontaneously between two opposing inaccessible landmarks (vertical black stripes) in an otherwise uniformly white surround. With their wings shortened, they are confined to an elevated circular disk $(8.5 \mathrm{~cm}$ diameter) surrounded by a water-filled moat. Tracks were recorded at a $5 \mathrm{~Hz}$ sampling rate with a video scanning device similar to that described by Bülthoff et al. (1982). Path lengths were calculated for five successive 3 min intervals of undisturbed walking and averaged over individuals. Moreover, walking speeds were calculated for every transition between the black stripes. For details of the setup and the analysis, see Strauß et al. (1992).

High-speed cinematography. One fly at a time with shortened wings was filmed at 200 frames/sec from above while walking on smooth, transparent graph paper in a "Buridan" arena (as described above). Step sizes, patterns, and durations of swing phases as a function of stepping period were obtained from frame-by-frame analyses. For a more detailed description, sec Strauß and Heisenberg (1990).

Fast phototaxis. The countercurrent apparatus first described by Benzer (1967) was used to obtain a relative measure of walking speed and readiness for walking under phototaxis conditions. Time allowance to reach the vial proximal to the light source was kept in such a range that 


\begin{tabular}{|c|c|c|c|c|c|}
\hline Name & $\begin{array}{l}\text { Abbrevi- } \\
\text { tion }\end{array}$ & Alleles & Map position & Cytological position & References \\
\hline agnostic & $a g n$ & $\mathrm{X} 1$ & $X-38.9$ & $11 \mathrm{~A} 7,11 \mathrm{~B} 9$ & $\begin{array}{l}\text { Savvateeva and Kamyshev, } 1981 \\
\text { Savvateeva et al., } 1992\end{array}$ \\
\hline central-body-defect & $c b d$ & $\begin{array}{l}\text { KS96 } \\
\text { KS171 } \\
\text { KS188 } \\
762 \\
2254\end{array}$ & $X-41$ & $11 \mathrm{~A} 1 ; 11 \mathrm{~A} 7$ & $\begin{array}{l}\text { Hanesch, } 1987 \\
\text { Heisenberg et al., } 1985\end{array}$ \\
\hline $\begin{array}{l}\text { central-complex-broad } \\
\text { central-complex-deranged }\end{array}$ & $\begin{array}{l}c c b \\
c c d\end{array}$ & $\begin{array}{l}\text { KS145 } \\
\text { KS135 }\end{array}$ & $\begin{array}{l}X-56.7 \\
X-15\end{array}$ & & $\begin{array}{l}\text { Heisenberg et al., } 1985 \\
\text { Hanesch, } 1987 \\
\text { Heisenberg et al., } 1985\end{array}$ \\
\hline central-brain-deranged & $c e b$ & $\begin{array}{l}849 \\
892\end{array}$ & $X-23$ & $7 \mathrm{~F} 1-2,8 \mathrm{~A} 4-5$ & Dorsch, 1985 \\
\hline central-complex & $\operatorname{cex}$ & KS181 & $X-43$ & $11 \mathrm{~A} 7,11 \mathrm{~B} 9$ & \\
\hline ellipsoid-body-open & $e b o$ & $\begin{array}{l}\text { KS263 } \\
678 \\
1041\end{array}$ & $X-3.8$ & & $\begin{array}{l}\text { Ilius et al., } 1992 \\
\text { Ilius, } 1992 \\
\text { Hanesch, } 1987 \\
\text { Heisenberg et al., } 1985\end{array}$ \\
\hline no-bridge & $n o b$ & KS49 & $X-12$ & $4 \mathrm{~F} 5,4 \mathrm{~F} 12$ & Strauß et al., 1992 \\
\hline
\end{tabular}

the number of successful flies was a function of this cycle duration. For each set of approximately 50 flies, five consecutive cycles were carried out with one fixed cycle duration. Time was varicd thercafter for another set of flies. According to Benzer (1967), the flies behave as independent individuals for the dimensions of the test apparatus used and the number of flies tested simultaneously. Statistically, the measurements have been treated like the respective number of single fly tests. Diameter of the vials: $15 \mathrm{~mm}$; the "toward light" part of the apparatus was reached after $72 \mathrm{~mm}$. The bottoms of the proximal row of vials had a distance of 30 $\mathrm{cm}$ from a $40 \mathrm{~W}$ fluorescent light (Osram-L, daylight deluxe).

Walking speed in negative geotaxis. One male fly at a time with shortened wings was shaken down to the bottom of a clear cylindrical Styrol fly container ( $49 \mathrm{~mm}$ diameter) that was covered with a black cap. The experiment was carried out on a black surface under uniform fluorescent light from above. Time was measured the fly needed from the start of the ascent at the vertical wall to a mark at a height of 82 $\mathrm{cm}$ above the ground. When a fly jumped onto the wall, paused during upward walking, or walked spirally the "run" was discarded. Ten valid walking speeds were obtained for each individual, and the fastest value was used to calculate a strain-specific average maximum upward walking speed from at least 10 flies.

Histology. The state of the central complex of every mutant fly was controlled after a behavioral test. Following embedding in paraffin (for mass histology, see Heisenberg and Böhl, 1979), brains were sliced in $7 \mu \mathrm{m}$ serial sections and inspected utilizing their autofluorescence.

\section{Results}

Fifteen mutant strains with structural defects in the $\mathrm{CC}$ were studied (Table 1). They belong to eight independent $\mathrm{X}$-linked genes. All but one had been isolated in F2 screens for structural defects in the brain using the mass histology technique of Heisenberg and Böhl (1979), following chemical mutagenesis. The mutant $a g n^{\mathrm{x}}$, in turn, had originally been known for its low spontaneous locomotor activity (Savvateeva and Kamyshev, 1981) and was identified as a structural mutant of the CC in the course of this study. Strains were compared to the wild types WT(B), C-S, O-R, and L-S.

\section{Structural defects}

The internal structure and location in and connections to the supraesophageal ganglion of the wild-type CC havc extensively been documented in Hanesch (1987) and Hanesch et al. (1989).
In all the mutants the $\mathrm{CC}$ is altered in its internal structure in one way or an other but is not completely missing. The typical strain-specific alterations are shown as schematic drawings in Figure 1. So far the structural alterations have not been studied in detail. In particular, virtually nothing is known about the effects of the mutations at the cellular or subcellular level. In order to indicate the range of structural defects among the 15 strains, we briefly describe them as they appear in the mass histology preparations. If not explicitly stated the ventral ganglion was not inspected.

In $n o b$ flies the protocerebral bridge (pb) is open in the middle. On either side of the midline glomeruli $1-3$ are missing. The adjacent glomeruli 4-6 are increased in size as if some of the material of the middle glomeruli had been displaced laterally. Glomeruli 7 and 8 have a normal appearance. In most animals the other neuropil substructures of the $\mathrm{CC}$, the fan-shaped body (fb), ellipsoid body (eb), and noduli, look normal. In some animals ( $5 \%$ in the present stock), however, the eb is open on its ventral side and the fb may have a cleft along the midline indicating a loosening of the neuropil in this region. No defects have been observed in other parts of the brain or ventral ganglion. For a somewhat more detailed account, see Strauß et al. (1992).

The gene $c b d$ has a profound effect on the formation and final structure of the CC. In all five alleles the structural defects are more severe than in any of the other strains. Interindividual variation is large. In most animals $\mathrm{fb}$ and $\mathrm{eb}$ are fused and the resulting mass of neuropil is fragmented in the middle ( $c b d^{\mathrm{Ks} 171}$, $c b d^{\mathrm{Ks} 188}, c b d^{2254}$, or even into several smaller masses dispersed over the central region of the protocerebrum $\left(c b d^{\mathrm{Ks} 96}, c b d^{762}\right)$. Also, the noduli often cannot be identified. Only the pb has a largely normal appearance. Occasionally, animals show a slight "thinning-out" of the $\mathrm{pb}$ in the region of the midline. The allele $c b d^{\mathrm{KS} 96}$ has the most severe $\mathrm{CC}$ defect, followed by $c b d^{762}$. The other three alleles are similar with respect to the $\mathrm{CC}$ but can be further distinguished by their pleiotropic defects.

In $20-40 \%$ of the animals of the strains $c b d^{\mathrm{Ks} 96}, c b d^{\mathrm{Ks} 171}$, and $c b d^{\mathrm{Ks} 188}$, the optic lobes are tilted forward and their internal 

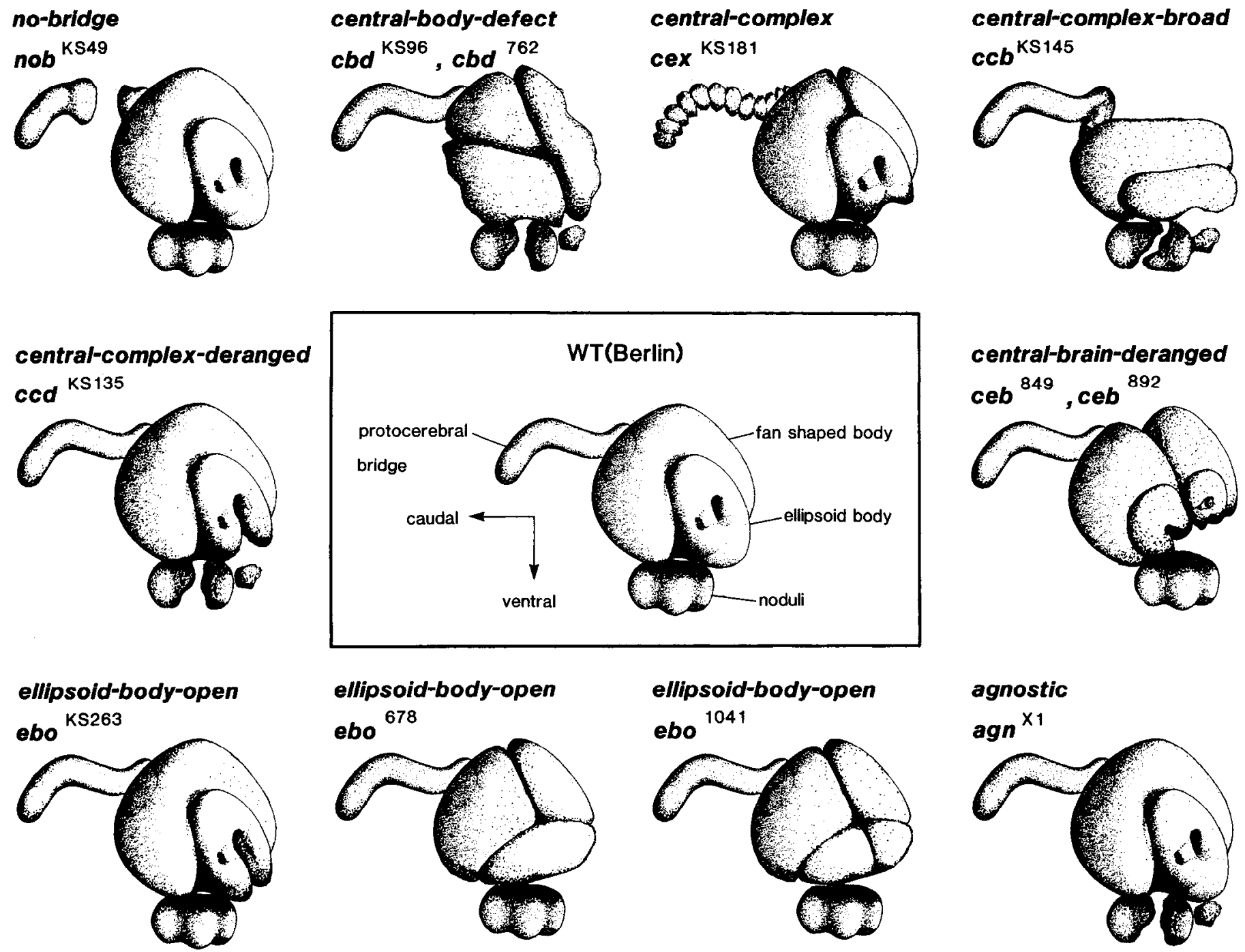

Figure 1. CC defects of the structural mutants used in this study: schematic representation of typical alterations as they appear in paraffin sections. A drawing of the wild-type CC (after Hanesch et al., 1989) is given in the center. In a dorsal-to-ventral order the CC is composed of the pb, the $\mathrm{fb}$, the eb, and the paired noduli. Out of five alleles of $c b d$, only the most severely affected alleles $K S 96$ and 762 are represented. In the alleles $\mathrm{KS171}, \mathrm{KS} 188$, and 2254 the fb typically has a median cleft, and in most flies eb and fb can be distinguished. For the alleles of ceb, a somewhat rare case is shown, where the symmetrical fragments of the eb form two small toroid-shaped ebs. For more detail about variable expressivity the reader is referred to the last paragraph of Results. Severely deformed fiber masses are not intended to be on scale.

structure is somewhat deranged. This impairment was observed only once in $c b d^{762}$ in a mild form and never in $c b d^{2254}$.

In the strain $c e x^{\mathrm{KS} 181}$, the $\mathrm{CC}$ defects seem subtle compared to $c b d$. The $\mathrm{pb}$ is less compact than in the wild type and the 16 glomeruli can easily be recognized. Along the first and possibly second glomeruli from the midline, the pb appears thinner. Also, the $\mathrm{fb}$ and ventral $\mathrm{eb}$ are affected along the midline: in many animals the $\mathrm{fb}$ has a cleft in the middle and may bend further forward around the eb than in the wild type. The eb may be ventrally open to varying degrees. The noduli occasionally are fragmented or fused. In all animals the CC defect is easily visible. No defects were observed in the optic lobes or in other parts of the brain.

In all animals of the three alleles of the gene $e b o$, the $\mathrm{CC}$ is deformed. As the name suggests, the common defect concerns the eb. The perfect doughnut shape of the wild-type eb may only show a small ventral cleft, but more frequently it has assumed the form of a kidney or horizontal bar. This bar often is dissociated into two separate horizontal fiber masses arranged symmetrically, and bending upward along the midline. The three alleles can be arranged according to the severity of their structural defects. The allele $e b o^{1041}$ is most strongly affected, followed by $e b o^{678}$ and $e b o^{\mathrm{Ks} 263}$. Minor defects in other parts of the $\mathrm{CC}$ are also apparent and in severity conform to the allelic order. In the present states of the stocks, no defects in the optic lobes are observed; however, right after isolation of the mutant the optic lobes of $e b o^{\mathrm{KS} 263}$ were also affected. Furthermore, a fourth allele, $e b o^{\mathrm{Ks} 234}$, not included in this study, has severe deformations of the optic lobes.

In the mutant central-complex-broad ${ }^{\mathrm{Ks} 145}\left(c c b^{\mathrm{Ks} 145}\right)$, the fb and eb are stretched along the transverse axis. The eb is opened and appears kidney shaped or as a horizontal bar. The expression is variable and overlaps wild type by about $20 \%$. Frequently the noduli are fragmented. In $50 \%$ of the animals the pb is slightly segmented, revealing its glomerular structure.

The structural phenotype of central-complex-deranged ${ }^{\mathrm{Ks} 135}$ 


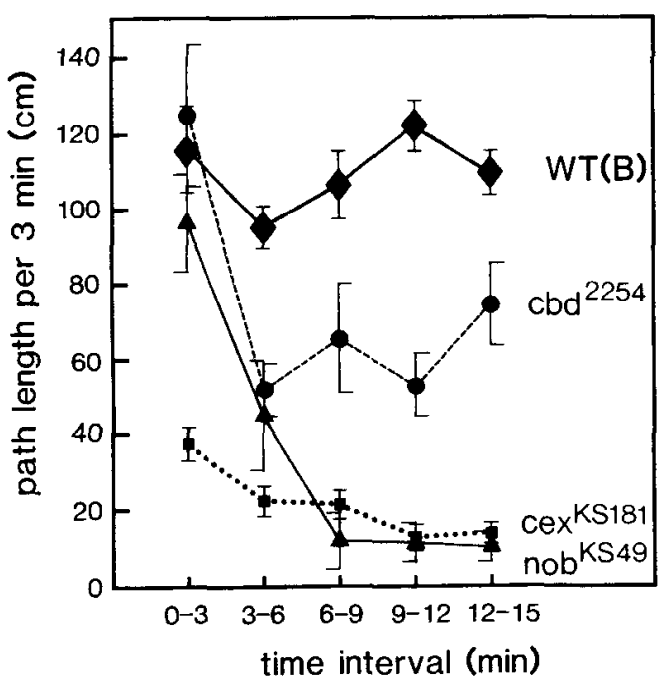

time interval (min)
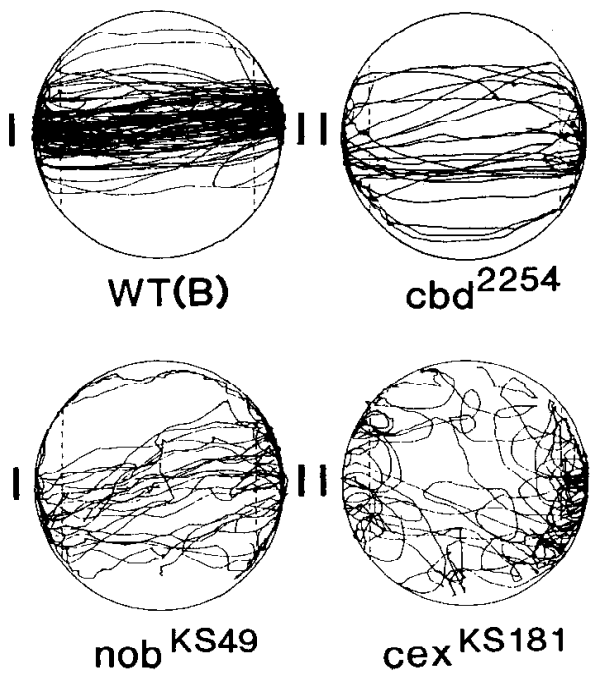

Figure 2. Mean time course of walking activity (mean path length per $3 \mathrm{~min}$ bin) in Buridan's paradigm for WT(B) and for the CC mutants $n o b^{\mathrm{KS} 49}, c b d^{2254}$, and $c e x^{\mathrm{KS} 181}$. Walking activity of the $\mathrm{CC}$ mutant strains decays significantly within $15 \mathrm{~min}$, each with a characteristic time course $\left[n_{\mathrm{WT} / \mathrm{B})}=11, n_{\mathrm{cbd}}=9\right.$, $n_{\text {nob }}=34$ (of which 23 were rejected: they did not complete a single walk between the landmarks), $n_{\mathrm{cex}}=14$ (of which 4 were rejected); error bars represent SEM]. For each strain typical single fly recordings of $15 \mathrm{~min}$ walking in Buridan's paradigm are given. Positions of the landmarks are indicated by vertical bars. Tracks of nob flies are less straight than those of WT(B). cex flies frequently return to the landmark they had just left. $\left(c c d^{\mathrm{Ks} 135}\right)$ resembles that of ebo mutants. It is, however, more strictly confined to the eb and a dissociation in two bilaterally symmetrical parts is not observed. In most animals the noduli appear slightly deformed or fragmented. In the present state of the stock, no defects are observed in the optic lobes, whereas right after isolation the optic lobes had been also deranged as in $c b d^{\mathrm{Ks} 96}$.

The two mutant alleles of the gene $c e b$ are structurally very similar and are described here together. They are distinguished from all strains mentioned so far in that they have a defect in the mushroom bodies in addition to the impairment of the $\mathrm{CC}$. About 400 Kenyon cell fibers miss the fiber bundle of the peduncle and form an extra lobe on the lateral side of the calyx. In the $\mathrm{CC}$ the defect is most prominent in the eb, which in half the animals is ventrally open as in the previously described mutants. In the other animals the fiber mass of the eb is divided into two parts along the midline. In some instances the two halves are reorganized into small doughnut-shaped structures symmetrical to the midline. The rest of the $\mathrm{CC}$ is affected in the usual manner: a median cleft in the fb of varying extent, a thinning of the pb along the midline in $50 \%$ of the cases, and an occasional slight derangement of the noduli.

In the mutant $a g n^{\mathrm{X}^{1}}$ the visible structural defect is confined to the noduli. In most animals of this stock, at the position of the noduli several small pieces of neuropil are found that are loosely connected among each other by thin fibers. They are locatcd at about the normal position between $\mathrm{fb}$, eb, and esophagus; however, in some animals they extend laterally as much as the width of the $\mathrm{CC}$. In some cases a large neuropilar mass without internal structure is found at the position of the noduli. In two animals a third nodulus of about half the normal size was found between the (normal) noduli and the eb.

\section{Walking activity (motivation for walking)}

In the behavioral studies all animals were killed after the experiment and subjected to paraffin histology as described. With few exceptions to be discussed below, no correlation was observed between the individual behavioral and structural defects.

Walking behavior was studied in three different paradigms: in an object fixation task (Buridan's paradigm; Götz, 1980), in fast phototaxis (Benzer, 1967), and in negative geotaxis. Influence of the mutations on walking activity is readily apparent in
Buridan's paradigm. When left undisturbed, flies of all four wildtype strains walk steadily between the two opposing landmarks during the 15 min test period (Fig. 2) and all individuals without exception explore both landmarks. In contrast, more than half of the mutant nob flies and about one-third of cex flies do not complete a single transition between the black bars. Nevertheless, such flies are able to walk and can fixate objects, for example, after being touched with a fly brush. Walking activity of initially walking individuals decays with strain specific time courses (Fig. 2). Decaying walking activity is further found in all five alleles of $c b d$ (for $c b d^{2254}$, see Fig. 2; timc courses of thc other alleles are not significantly different from each other; in $c b d^{762}$ flies the same time course is found on a lower average activity level; Fig. 3). A similar decay is observed with $a g n^{\mathrm{x} 1}$, $e b o^{\mathrm{KS} 263}, c c b^{\mathrm{KS} 145}$, and both alleles of $c e b$. Hence, a total of seven out of the eight genes affect the time course of walking activity. Even in flies of the remaining gene $c c d^{\mathrm{KS} 135}$, a downward trend in walking activity during the $15 \mathrm{~min}$ period in Buridan's paradigm is apparent, particularly in females (data not shown). Only the two strains $e b o^{678}$ and $e b o^{1041}$, in contrast to the allele $e b o^{\mathrm{KS} 263}$, maintain a uniform walking activity during the whole test period.

Decay of walking activity is not an unspecific side effect of structural brain defects. This is demonstrated by the steady walking activity of mutants with structural defects in the optic lobes such as optomotor-blind ${ }^{H 31}\left(\mathrm{omb}^{\mathrm{H} 31}\right)$ and small-opticlobes ${ }^{\mathrm{Ks} 58}$ (sol ${ }^{\mathrm{Ks} s 8}$; Fig. 3) or by a wild-type walking activity of the structural mutant mushroom-bodies-deranged ${ }^{\mathrm{KS} 65}$ in another locomotor activity test (Hanesch, 1987).

To distinguish a lack of activity from physical exhaustion, $n o b$ flies were taken out of Buridan's paradigm 6 min after they had been placed in the arena and were immediately brought back in with the initial handling procedure (Strauß et al., 1992). In the consecutive $15 \mathrm{~min}$, their time course of walking activity, average walking speed, and mean total track length were indistinguishable from those of the original experiment, thus ruling out exhaustion.

\section{Walking speed}

In Buridan's paradigm, flies of 14 alleles (8 independent genes) walk more slowly (mean and maximum) than the fastest wild type WT(B) (for average walking speeds, see Fig. 4; maximum 


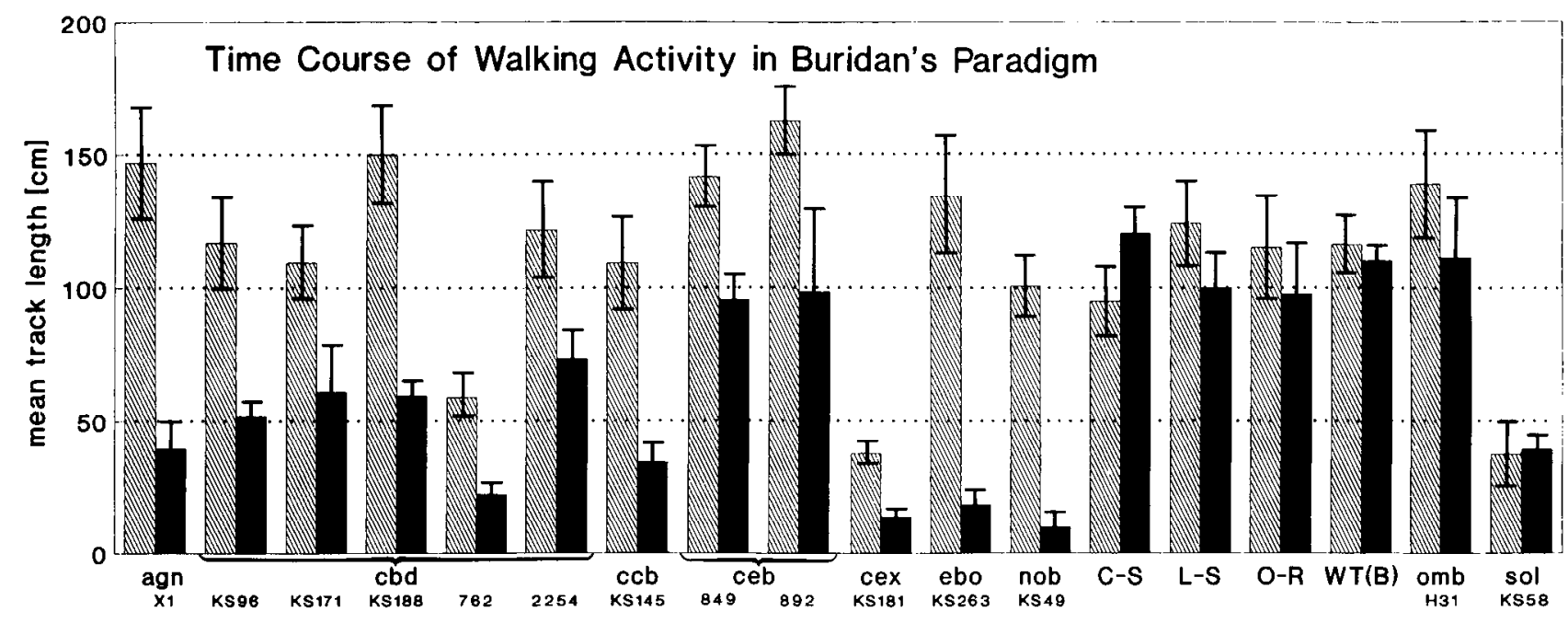

Figure 3. Strain-specific time courses of walking activity in "Buridan's paradigm." Walking activity is represented by the mean distances \pm SEM covered during the first $3 \mathrm{~min}$ (hatched bars) and during the $3 \mathrm{~min}$ interval from minutes 13-15 (solid bars). Twelve out of 15 alleles (seven independent genes) show significantly and steadily decaying walking activity. In contrast, all wild-type strains and both structural mutants of the visual system tested in this respect walk steadily during the $15 \mathrm{~min}$ recording period. While all flies of the wild-type strains completed more than one transition between the landmarks, not all mutant flies do. Nonwalkers are excluded from the mean value and are counted separately. The following numbers of male flies contributed to the mean: $a g n^{\mathrm{XI}}, 9$ of $10 ; c b d^{\mathrm{KS} 96}, 9$ of $10 ; c b d^{\mathrm{KS} 171}, 10 ; c b d^{\mathrm{KS} 188}, 10 ; c b d^{762}, 8$ of $10 ; c b d^{2254}, 8$ of 9 ; $c c b^{\mathrm{KS} 145}, 13 ; c e b^{849}, 10 ; c e b^{892}, 8 ; c e x^{\mathrm{KS} 181}, 10$ of $14 ; e b o^{\mathrm{KS} 263}, 11$ of $12 ; n o b^{\mathrm{KS} 49}, 11$ of 34 ; wild-type strains: C-S, 14; L-S, 10; O-R, 10; WT(B), 22; mutants of the visual system: $o m b, 6 ;$ sol, 10 . Only three CC-defective alleles show constant walking activity and are not included in this figure: $e b o^{678}, 9$ of 10 ; $e b o^{1041}, 9$ of $10 ; c c d^{\mathrm{KS} 135}, 10$ (see Walking activity, above, for decay in $c c d^{\mathrm{KS} 135}$ females).

speed, $4.2 \mathrm{~cm} / \mathrm{sec}$ ). For the six strains $a g n^{\times 1}$ (maximum speed, $2.4 \mathrm{~cm} / \mathrm{sec}), c c b^{\mathrm{KS} 145}(2.9 \mathrm{~cm} / \mathrm{sec}), c c d^{\mathrm{KS} 135}(2.6 \mathrm{~cm} / \mathrm{sec}), c e x^{\mathrm{KS} 181}$ $(1.6 \mathrm{~cm} / \mathrm{sec}), e b o^{678}(2.4 \mathrm{~cm} / \mathrm{sec})$, and $n o b(2.8 \mathrm{~cm} / \mathrm{sec})$, differences are also significant compared to the slowest of the wildtype strains under study, C-S (maximum walking speed, $3.2 \mathrm{~cm} /$ sec).

We wondered whether vanishing motivation for walking and low walking speed in the mutants is specific for Buridan's paradigm or might be a general property of walking independent of the respective experimental procedure. Comparing average strain-specific maximum walking speeds in vertical upward walking (negative geotaxis), six of seven nonallelic CC mutant strains tested were significantly slower than WT(B) and C-S flies (Fig. 5). Interestingly, the two mutant alleles of ceb that have only a small decrement in walking activity over $15 \mathrm{~min}$, and of which one has the highest mutant score in walking activity in Buridan's paradigm, also score the highest in this test. At the low end, cex $\mathrm{KS181}^{\mathrm{K}}$ is the most severely affected strain in both experiments. Among the various alleles of the same gene, however, surprising deviations are observed. For instance $c e b^{892}$ is significantly faster than $c e b^{849}$ in Buridan's paradigm but slower in upward walking. Similar differences are found among the three alleles of ebo. These allele differences at present cannot be explained.

\section{Fast phototaxis}

As a further experiment for measuring walking activity and specd, fast phototaxis was studied in eight nonallelic CC mutants and in two wild-type strains (Fig. 6). WT(B) reaches a maximum value of $83 \pm 4 \%$ of all possible transitions toward light at an optimum cycle duration of $15 \mathrm{sec}$ (see Materials and Methods). Longer cycle times do not improve on that value, since the fastest flies already tend to return to the vials distal from light. The wild-type strain C-S-walking more slowly than WT(B) in
Figure 4. Mean walking speed \pm SEM for transitions between the landmarks recorded over $15 \mathrm{~min}$ per fly in Buridan's paradigm. Flies of all strains but $c e b^{892}$ walk more slowly when compared to their original background, WT(B). Six strains, agn, $c c b, c c d$, cex, $e b o^{678}$, and $n o b$, walk even more slowly than the slowest wild-type strain, C-S. Same set of data as indicated for Figure 3.

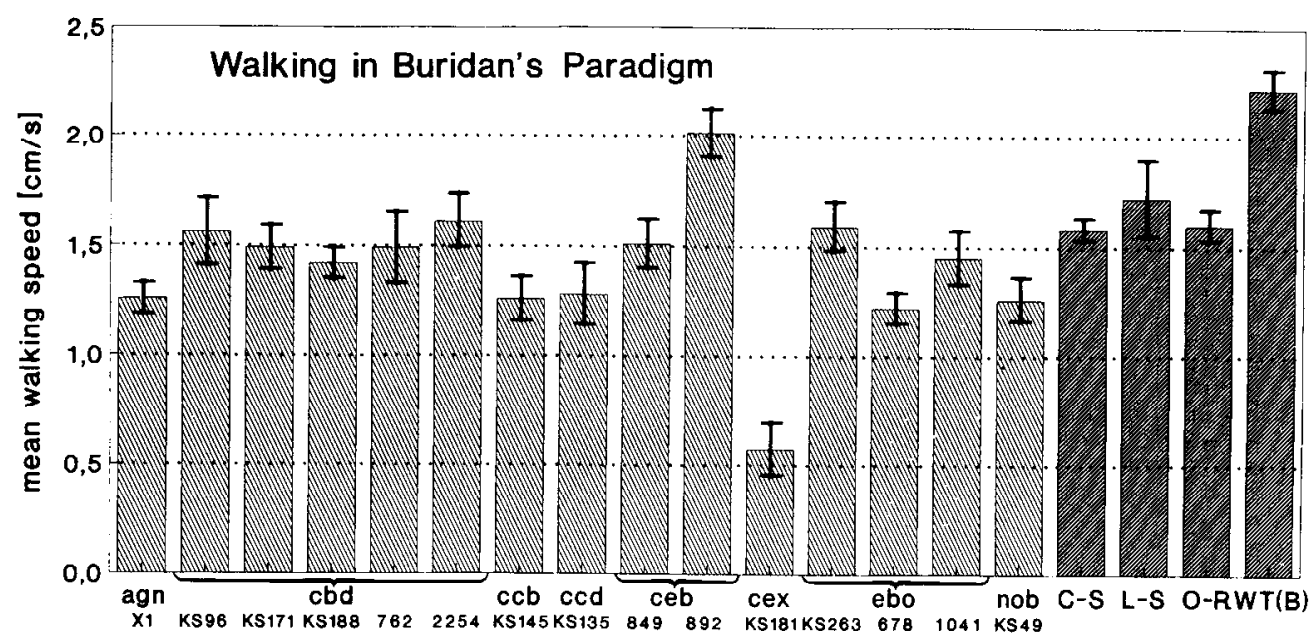




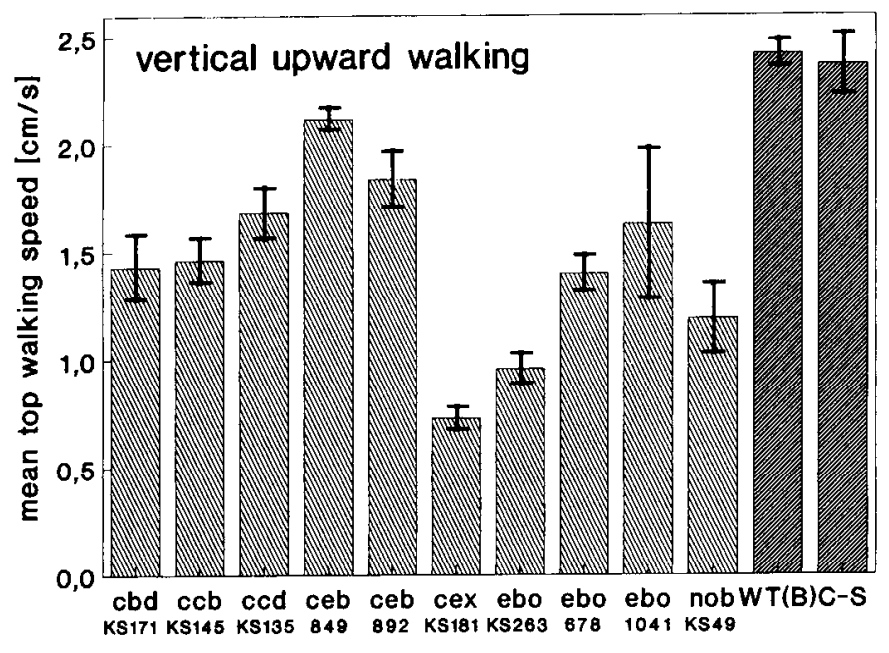

Figure 5. Mean strain-specific top speed (the definition is given in Materials and Methods) in vertical upward walking (negative geotaxis). The following numbers of male flies contributed to the mean: $c b d^{\mathrm{Ks} 171}$, $11 ; c c b^{\mathrm{KS145}}, 10 ; c c d^{\mathrm{KS} 135}, 10 ; c e b^{849}, 10 ; c e b^{892}, 10 ; c e x^{\mathrm{KS} 181}, 10$ (tested over $30 \mathrm{~mm}$ since no individual covered the original test distance of $82 \mathrm{~mm}$ without pausing); $e b o^{\mathrm{KS} 263}, 10 ; e b o^{678}, 10 ; e b o^{1041}, 10 ; n o b^{\mathrm{KS} 49}$, 12 of 20; C-S, 10; WT(B), 10.

Buridan's paradigm - is faster in this test and reaches its peak transition value already at cycle durations of $10 \mathrm{sec}$.

Of the eight CC mutant strains tested, only agn and $c c d$ flies are entirely normal in fast phototaxis, indicating that they can overcome their reduced speed displayed in Buridan's paradigm and negative geotaxis. In contrast, the delayed reaction of $c b d \mathrm{ks} 171$ flies is in quantitative agreement with their $30 \%$ slower walking speed as compared to WT(B). They reach the same transition scores as the wild type if they are allowed 50\% longer cycle times.

The behavior of $c e b$ flies is best described as a rapid loss of the aroused condition, which in this test is caused by shaking the flies down at the beginning of each cyclc. For both $c e b$ alleles, longer cycle durations do not improve on the number of tran- sitions. Instead, flies tend to distribute equally over proximal and distal parts of the test vials in a seemingly "relaxed" state.

Fast phototaxis is greatly suppressed in $c c b^{\mathrm{KS} 145}$, $c e x^{\mathrm{KS} 181}$, $e b o^{\mathrm{KS} 263}$, and $n o b^{\mathrm{K} \$ 49}$. Accounting for their known slower walking speeds by prolonging cycle durations, respectively, did not yicld normal transition numbers. Toward longer cycle durations, the number of transitions under fast phototaxis conditions was only slightly higher than the number of transitions under uniform illumination (see cex "diffusion" in Fig. 6). In these strains, including the two alleles of $c e b$, other aspects of fast phototaxis besides walking speed must be affected by the mutations. (Note that none of the mutants are simply blind as, for instance, the performance in Buridan's paradigm and optomotor tests show.)

Each of the strains has its idiosyncrasies. Straightness of walking, for instance, is impaired in nob flies. Their leg coordination tends to be disturbed during turning and start/stop maneuvers (Straußet al., 1992). In Buridan's paradigm, cex flies often return to the ipsilateral landmark (Fig. 2). Flies of $e b o^{678}$ frequently walk perpendicular to the axis connecting the two landmarks. Which of these behavioral traits depend upon defects in the $\mathrm{CC}$ is not clear.

\section{Leg coordination}

Three strains, the mutants $n o b^{\mathrm{KS} 49}, c b d^{2254}$, and $c e x^{\mathrm{KS} 181}$ were studied in more detail. Walking patterns were recorded at 200 frames/sec to assess leg coordination, step size, and stepping frequency. In nob this technique reveals a striking reduction in step lengths for walking speeds above $v=1.5 \mathrm{~cm} / \mathrm{sec}$ (Fig. 7a). These small steps are performed during swing phases of almost normal duration.

To test the specificity of this trait for the nob mutation, the dependence of step length upon period was analyzed in two strains with a wild-type CC but walking more slowly than WT(B) (Fig. $7 b, c)$. The characteristic reduction in step length at high walking speed of $n o b$ is not observed for C-S or $s o l^{\text {Kss }}$ flies.

In $c b d^{2254}$ mutant flies also a striking reduction in step lengths is observed. In contrast to nob, however, this is due to swing phases shortened proportionally. As was shown with mosaic

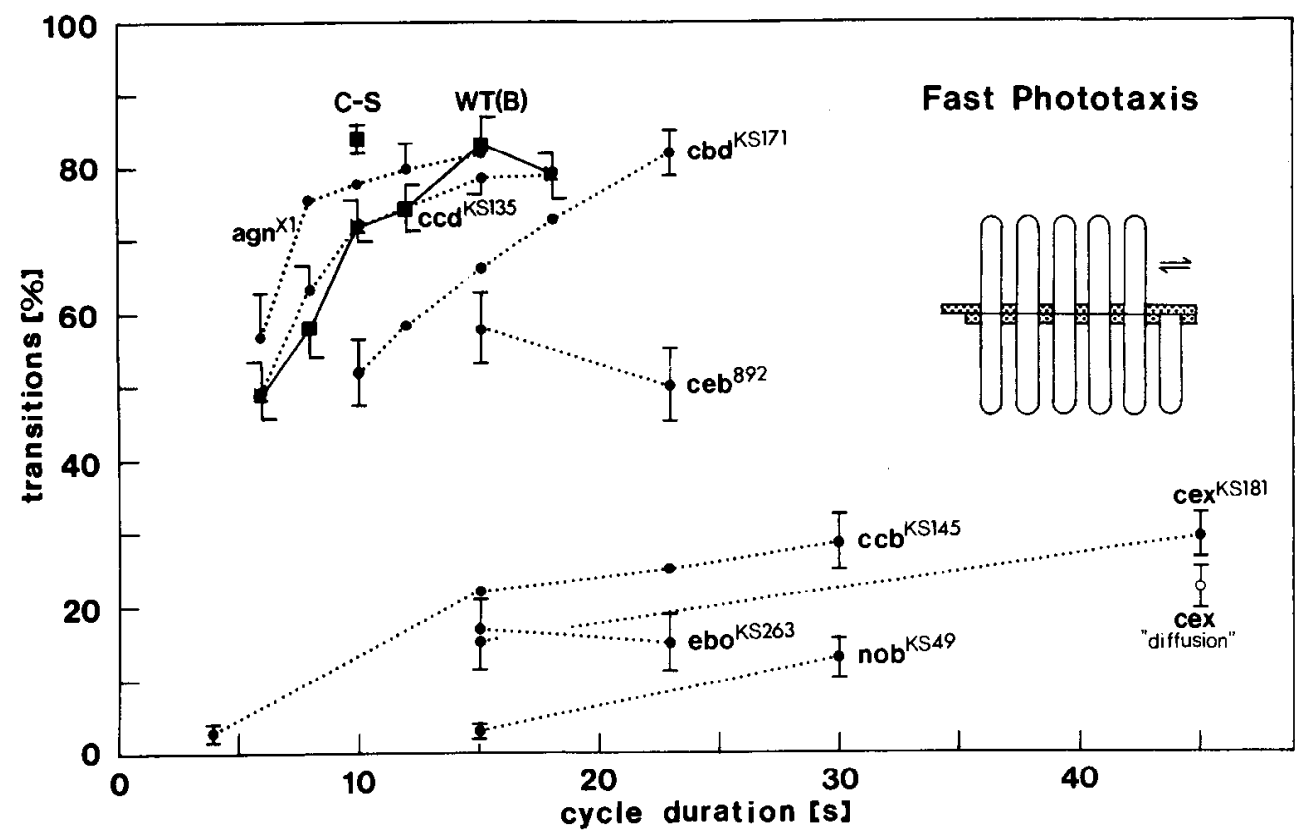

Figure 6. Fast phototaxis: transitions toward light versus cycle duration given to reach the vials proximal to the light. Number of transitions is expressed in percentage of the total possible number of transitions, that is, 5 cycles $\times$ number of flies per test. Each data point represents one or two sets of 50 flies tested in five consecutive cycles of constant duration. Cycle duration was varied thereafter for a fresh set of flies. Error bars denote SEMs. cex "diffusion," Experiment carried out under uniform illumination. Male flies of the CC-mutant strains $a g n^{\mathrm{X} 1}, \mathrm{cbd}^{\mathrm{KS} 171}, c c b^{\mathrm{KS} 145}$, $c c d^{\mathrm{KS} 135}, c e b^{892}, c e x^{\mathrm{KS} 181}, e b o^{\mathrm{KS} 263}$ and $n o b^{\mathrm{KS} 49}$, and of the wild-type strains WT(B) and C-S (only peak transition rate shown) were used. 


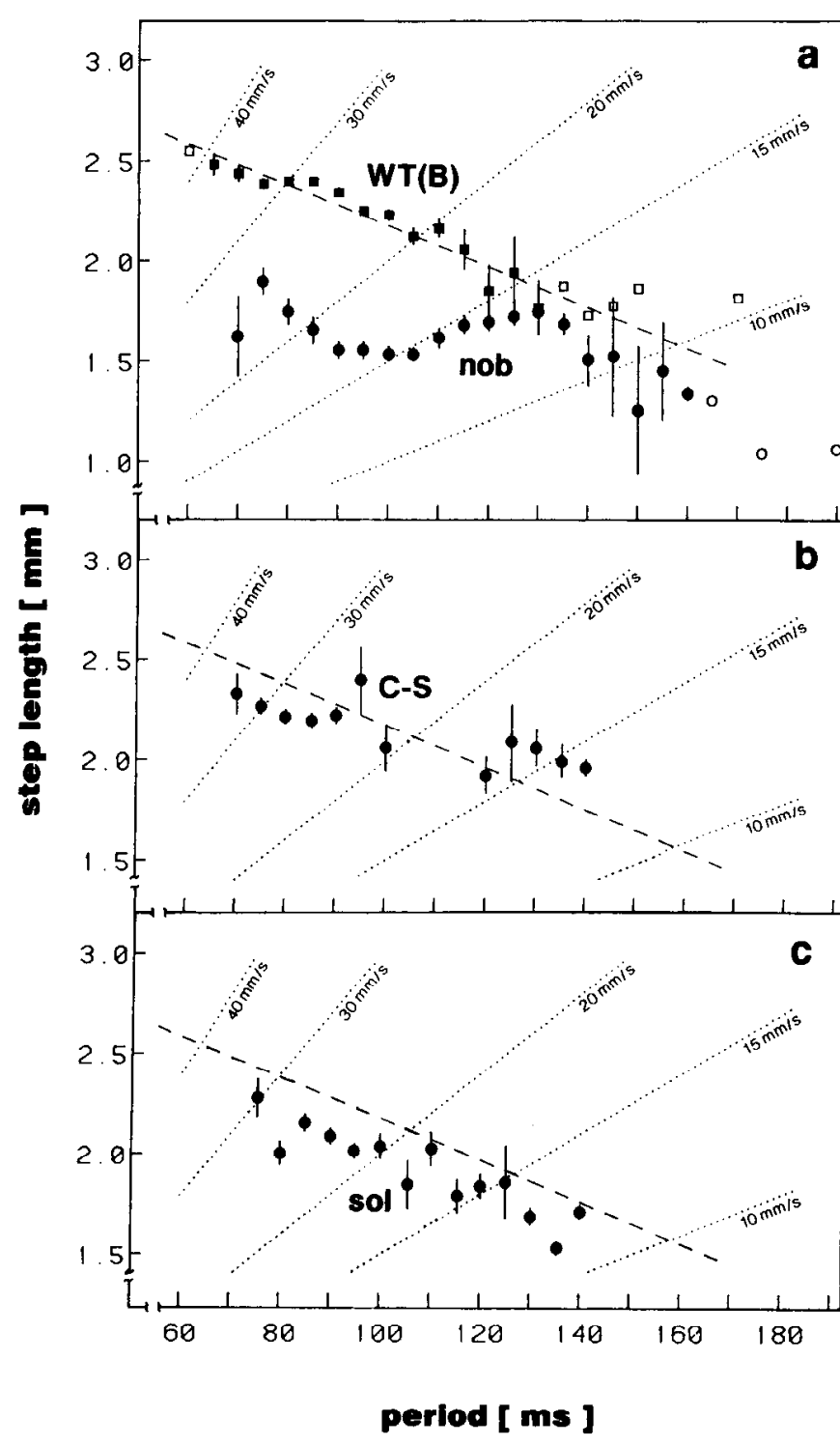

Figure 7. Mean dependence of step length upon period during straight walking for $10 \mathrm{WT}(\mathrm{B})$ and 6 nob flies (a). Solid symbols represent mean step lengths for a given mean period averaged over all legs $( \pm$ SEM where this is larger than the symbol). Open symbols denote single steps. Total number of steps is 1736 for WT(B) and 509 for nob. The dashed lines in $a, b$, and $c$ indicate the regression line through the WT(B) data. Dotted lines connect combinations of periods and step lengths leading to the same walking speed (isotaches). To prove the specificity of the $n o b$ behavior, samples of each 183 steps were taken from walks with extremal walking speeds from two C-S wild-type flies $(b)$ and from two sol $\mathrm{KSss}^{\mathrm{s}}$ mutant individuals $(c)$. Both strains walk more slowly than WT(B) flies, but do so close to the WT(B) characteristics: WT(B), $2.22 \pm 0.09$ $\mathrm{cm} / \mathrm{sec}, 4.2 \mathrm{~cm} / \mathrm{sec}$ at maximum; C-S, $1.58 \pm 0.05 \mathrm{~cm} / \mathrm{sec}, 3.2 \mathrm{~cm} / \mathrm{sec}$ at maximum; sol ${ }^{\mathrm{Ks} s 8}, 1.35 \pm 0.15 \mathrm{~cm} / \mathrm{sec}, 3.1 \mathrm{~cm} / \mathrm{sec}$ at maximum; $n o b^{\mathrm{KS} 49}, 1.26 \pm 0.10 \mathrm{~cm} / \mathrm{sec}, 2.6 \mathrm{~cm} / \mathrm{sec}$ at maximum (data in $a$ from Strauß et al., 1992).

flies (see below), this phenotype does not relate to the mutant state of the CC. In cex ${ }^{\mathrm{Ks} 181}$ flies, the exceptionally slow average and maximum walking speeds are due to aberrations in the temporal sequence of swing phases. Again, this behavioral defect seems to be unrelated to the structural defect in the CC.

\section{Mosaic analysis}

Although in the mutants the structural defects as revealed by conventional paraffin histology are largely confined to the $\mathrm{CC}$, structures or physiological functions in other parts of the fly may also be impaired by the mutations. For a closer correlation between behavioral and structural defects, gynandromorph flies with a mutant brain and a wild-type thorax were compared to individuals with a wild-type brain and a mutant thorax (mutant cuticle marked with $s n$ or $f$ ). Mutant strains $c b d^{2254} s n^{3}, c e x^{\mathrm{Ks} 181} f$, $y w^{\mathrm{a}} \mathrm{nob}^{\mathrm{KS} 49} f$, and $n o b^{\mathrm{KS} 49} \mathrm{sn}^{3}$ (Fig. 8) were studied. In all cases the focus for the time course of walking activity was located in the brain and full correlation with the respective state of the $\mathrm{CC}$ was found (assessed by histology for each individual after the test). Moreover, two nob gynandromorphs with an incomplete structural defect of the pb showed intermediate behavioral values for walking activity and its decay.

Analysis of the nob mosaics places the focus for walking speed in the brain and, again, full correlation with the state of the pb is found (Fig. 9; the contribution of the two mosaic individuals with partially defective $\mathrm{pb}$ is indicated by open bars).

There is no evidence so far for an influence of the $\mathrm{CC}$ on the temporal order of swing phases during straight walking, in agreement with the idea that this pattern is generated in the thorax. The focus for the aberrations in the temporal sequence of swing phases in cex flies probably resides in the ventral ganglion, as gynandromorph analysis reveals. Also, the focus for reduced step length in $c b d^{2254}$ does not coincide with the CC. However, a role of the $\mathrm{CC}$ in turning and start/stop maneuvers is suggested by aberrations found in the pattern of swing phases of nob flies during such maneuvers. Since these episodes significantly influence walking speed the foci for the corresponding defects may also reside in the $\mathrm{CC}$.

\section{Variable expressivity}

In most structural brain mutants with variable expressivity of the structural defect, the strengths of the structural and behavioral defects are poorly correlated (for a possible explanation, see Heisenberg et al., 1985; Brunner et al., 1991). While this applies to the various alleles of $c b d$ and $e b o$, it seems to be different for $n o b$ and $c c b^{\mathrm{Ks} 145}$. Among 159 nob flies studied in Buridan's paradigm in a different context, two individuals with partially reverted pb were found. They had intermediate scores for both walking speed and activity (data not shown). Likewise for $c c b^{\mathrm{KS} 145}$ flies, walking speed and activity appear to be negatively correlated with the severity of the structural defect in the CC (Fig. 10).

\section{Discussion}

The study of 15 strains with defects in eight different genes affecting the structure of the $\mathrm{CC}$ demonstrates its influence on walking activity, walking speed, leg coordination, and possibly "straightness of walking." All were found to be impaired either in a general or a paradigm-dependent manner.

Flies of all eight genes (13 of 15 alleles; $c c d^{\mathrm{Ks} 135}$ females only) show decaying walking activity in "Buridan's paradigm." In all strains studied in detail ( $\left.n o b, c b d^{2254}, c e x\right)$ the focus of the activity phenotype is located in the brain and full correlation with the respective state of the $\mathrm{CC}$ is found. What further adds to the evidence is the high correlation between the degree of the behavioral impairment and the severity of the structural $\mathrm{CC}$ defect in two strains. The decay is not an unspecific side effect of structural brain defects as has been demonstrated by the steady walking activity of structural mutants of the visual system, two alleles of the CC mutant $e b o$, and of mushroom body mutants. It has been proven in the case of nob, that vanishing 

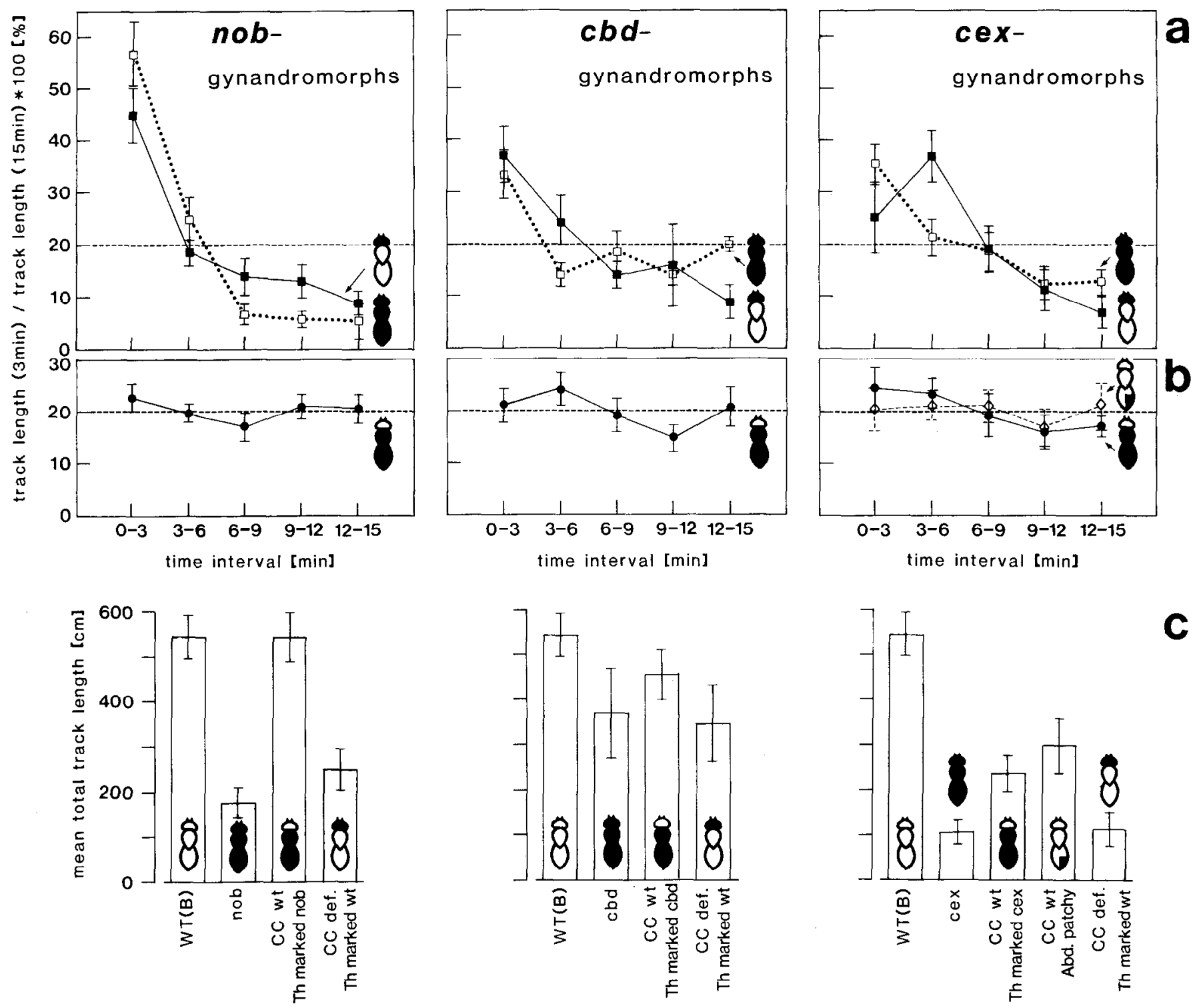

Figure 8. Results of gynandromorph analyses carried out on $n o b^{\mathrm{KS} 49}, c b d^{2254}$, and $c e x^{\mathrm{ks} 181}$. In $a$ the time courses of walking activity of mosaic flies with a strain specific defective $\mathrm{CC}$ and a thorax $(T h)$ marked for the wild-type genotype are compared to the time courses of the respective mutant strains under study (the pictogram flies indicate mutant tissue in black). Note that time courses are given in relation to the 15 min total walking activity of a test group, that is, constant walking activity yields five times a value of $20 \%$ (dashed lines; constant walking activity was found in all four different wild-type strains tested). The time courses of walking activity of the test groups with wild-type CCs and thoraxes externally marked for mutant genotypes are shown in $b$. Mean total track lengths of all test groups are given in $c$. All error bars denote SEMs. The state of the CC was controlled histologically for every mosaic and mutant individual after the behavioral test. The genotype of the ventral ganglion could only indirectly be assessed from the distribution of the external markers. The decay in walking activity caused by a cex-defective CC can be separated from an influence of cex on leg coordination, leading to a reduction in walking speed. Constant walking activity was observed whenever the cex gene did not affect structures of the CC ("thorax group," "patchy abdomen group"). Errors in leg coordination concomitant with a reduction in walking speed were found in the "thorax group" but also in four of nine "patchy abdomen" individuals and in two individuals of the CC-defective group. This variation is in agreement with fate map data (Kankel and Hall, 1976). Mean total track lengths of the cex "thorax group" and the "patchy abdomen group" are depressed proportionally to their reduction in average walking speed. Number of mosaic flies included in the nob study: mosaics with defective CC and wild-type thorax, 13 marked with $s n^{3}$ (5 without a walk), and 11 marked with $y w^{\mathbf{a}} f$ (two without a walk, two with one walk); intact $C C$ and thorax marked for $n o b, 13$ marked with $s n^{3}$, and 11 marked with $y w^{2} f . c b d^{2254}$ study (marker $s n^{3}$ ): defective $C C$ and wild-type thorax, 9; wild-type $C C$ and thorax marked $c b d, 9$. cex study (marker $f$ ): defective CC and wild-type thorax, 9 (2 without a walk); wild-type $C C$ and mutant thorax, 8; wild-type $C C$, a wild-type marked thorax, and patches of $c e x f$ tissue on the abdomen, 9 . Wild-type (WT(B)) and mutant $(c b d, c e x)$ data from Figures 2 and 3 are included for comparison.

activity is not due to physical exhaustion. The nonwalking nob and cex flies in Buridan's paradigm are considered to represent the extreme form of the same activity phenotype, since such flies can be stimulated to walk. Moreover, reduced or rapidly decaying walking activity is also apparent in fast phototaxis in five of eight CC mutant strains studied in that respect.

In Buridan's paradigm, 14 mutant alleles of seven independent genes walk more slowly (mean and maximum) than the 


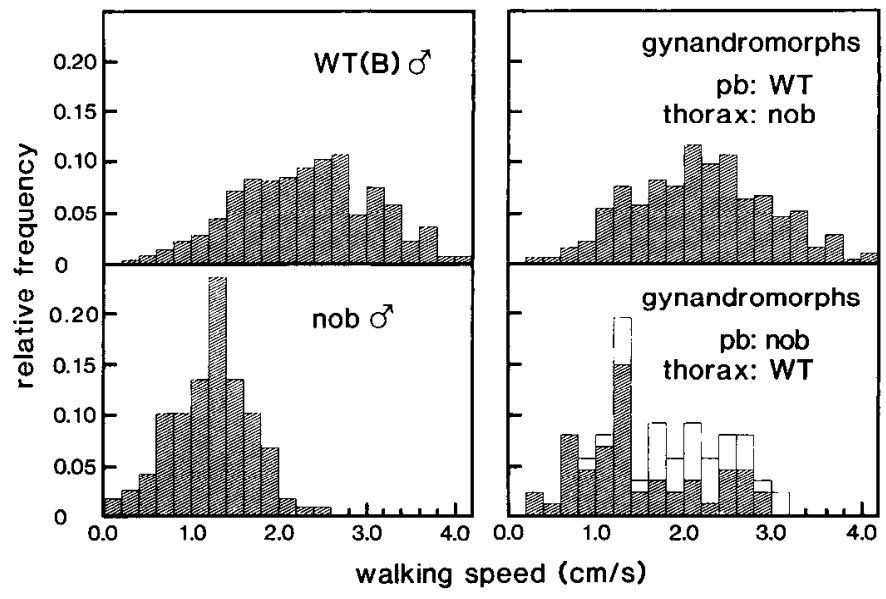

Figure 9. Distribution of walking speeds for walks between the landmarks in Buridan's paradigm for two groups of nob gynandromorphs in comparison to WT(B) and mutant nob flies (same set of nob $s n^{3}$ gynandromorph data as in Fig. 8). Stretches of fly tracks between two virtual lines (indicated as dashed lines in the recordings of Fig. 2) are taken as "walks" if the fly had crossed both lines (distance, $6.6 \mathrm{~cm}$ ). Actual track lengths are used to calculate average walking speeds for individual walks. Values are classified with a bin size of $0.2 \mathrm{~cm} / \mathrm{sec}$. Compared to WT, nob flies show reduced maximum and average walking speeds. Walking speeds of gynandromorphs correspond to the respective states of the pbs and are independent of the genotype of the thorax. A significant difference in mean walking speeds is given between the two groups of gynandromorphs but none between WT flies and the $\mathrm{WT}-\mathrm{pb} / n o b$-thorax group and none between $n o b$ and the $n o b$-pb/WTthorax group. The contribution of two gynandromorphs with incomplete $n o b$-defective $\mathrm{pb}$ is indicated as open bars. Their intermediate walking speed provides further evidence for the correlation with the state of the $\mathrm{pb}$.

fastest wild-type strain, WT(B). For six nonallelic CC mutant strains differences are also significant compared to C-S, the slowest of the wild-type strains tested. Comparing strain-specific average maximum walking speeds in negative geotaxis, six of seven nonallelic $\mathrm{CC}$ mutant strains examined are significantly slower than both WT(B) and C-S flies.

Influence of the $\mathrm{CC}$ on parameters of leg coordination is apparent in mosaic nob flies: their reduced average and maximum walking speeds are fully correlated with the defective state of the $\mathrm{pb}$. Slow walking, in turn, is due to shorter steps performed during swing phases of almost normal duration. To the contrary, no indication is found of an involvement of the $\mathrm{CC}$ in setting up the basic stepping rhythm during straight walking. The focus for the crrors in leg coordination of the mutant cex resides in the thorax. The finding is in agreement with the current understanding of insect walking, according to which the pattern-generating network for walking is localized in the ventral ganglion (Bässler, 1983; Graham, 1985).

Nevertheless, influences of insect head ganglia on walking are very specific. This has been demonstrated, for example, by Graham (1979) in decerebrate stick insects. While walking was fully coordinated, Graham identified subtle changes in timing such as a longer average step period or momentary pauses during stance phases. Kien (1983) delivered extracellular microstimulation to circumesophageal and neck connectives in tethered walking Schistocerca. She could evoke different reproducible types of walking or turning at 73 loci, of which 10 were common to both connectives and 37 were found only in the circumesophageal connectives. The elicited walking patterns differed in direction, speed, step length, or positioning of the legs relative to the body.

Our results show that the $\mathrm{CC}$ is at least one important site within the supraesophageal ganglion for such fine-tuning of walking behavior. In all 15 strains the structural defects are highly specific to the $\mathrm{CC}$, at least at the level of the light microscope. The mosaic studies further confine the focus for the behavioral impairments to the central brain and make any site other than the $\mathrm{CC}$ unlikely. This assignment concerns the regulation of walking activity, walking speed, and complex walking maneuvers. Studies on other types of behavior suggest that the $\mathrm{CC}$ may regulate behavioral activity in general (for flight control, Strauß et al., 1992; Ilius et al., 1992; for escape behavior, Strauß et al., 1992).

The conclusions are in agreement with early electrostimulation and coagulation experiments (Huber, 1959, 1960; Otto, 1971). These authors have shown that both normal and abnormal patterns of behavior such as walking, singing, or preening can be evoked or suppressed, respectively, in the CC.

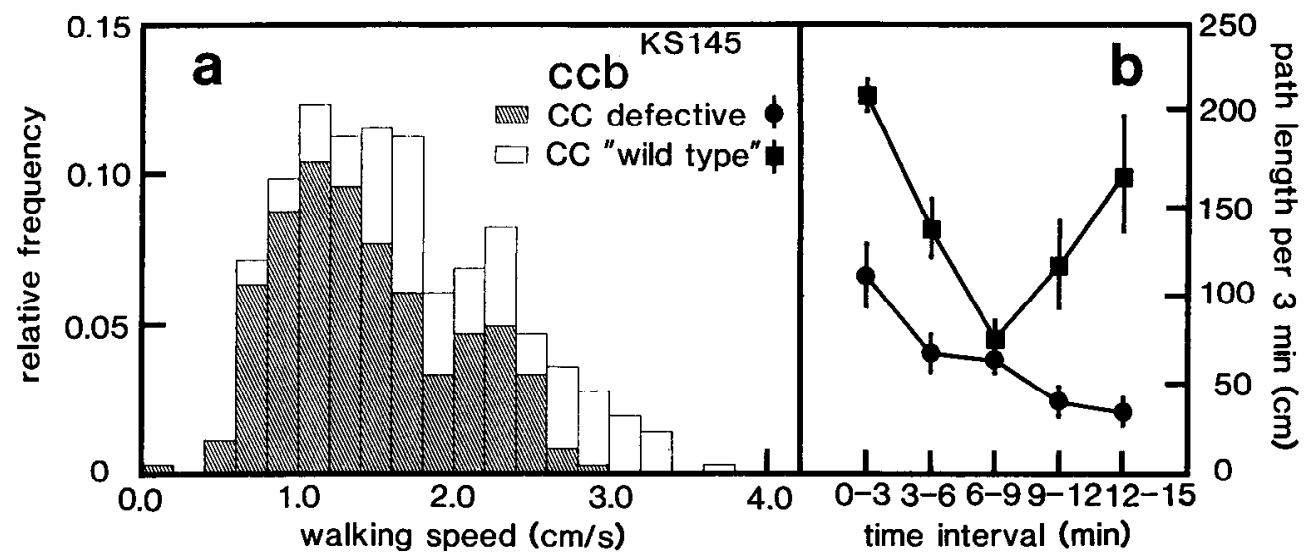

Figure 10. Correlation between parameters of walking behavior and the variably affected structure of the $\mathrm{CC}$ in $c c b^{\mathrm{Ks} 145}$ male flies (the strain is known for its variable expressivity). Three out of 16 mutant individuals were histologically identified to have had almost wild-type CCs. Data of those individuals were treated separately in the figure ( $C C$ ' wild type'). In a distribution of walking spccds for walks between the landmarks in Buridan's paradigm (a), all walks faster than $2.9 \mathrm{~cm} / \mathrm{sec}$ are due to the $C C$ "wild type" group (mean walking speed, $2.1 \pm 0.1 \mathrm{~cm} / \mathrm{sec}$; CC-defective group, $1.5 \pm 0.1 \mathrm{~cm} / \mathrm{sec}$ ). While the walking activity of the CC-defective individuals is steadily decaying $(b)$, the respective $C C$ "wild type" group obeys no clear trend and total activity (mean total track length: $668 \pm 173 \mathrm{~cm}$ ) is well above the CC-defective group (mean total track length, 310 $\pm 50 \mathrm{~cm})$. 
The demonstration of a higher motor control center in the arthropod brain is of considerable importance for a general understanding of brain function. Vertebrate and arthropod brains alike seem primarily to be concerned with the control, organization, and planning of behavior. Presumably, the pattern generator for undisturbed straight walking is located in the ventral ganglion; the readiness to use it, the vigor of the strides, and probably modifications of the pattern (e.g., for turning) are contributed by the $\mathrm{CC}$ in the brain.

\section{References}

Bässler U (1983) Neural basis of elementary behavior in stick insects. In: Studies of brain function, Vol X (Braitenberg V, ed). Berlin: Springer.

Benzer S (1967) Behavioral mutants of Drosophila isolated by countercurrent distribution. Proc Natl Acad Sci USA 58:1112-1119.

Brunner A, Wolf R, Pflugfelder GO, Peock B, Heisenberg M (1992) Mutations in the proximal region of the optomotor-blind locus of Drosophila melanogaster reveal a gradient of neuroanatomical and behavioral phenotypes. J Neurogenet 8:43-55.

Bülthoff $H$, Götz KG, Herre M (1982) Recurrent inversion of visual orientation in the walking fly. J Comp Physiol [A] 148:471-481.

Dorsch M (1985) Histologische und verhaltensphysiologische Untersuchungen einer strukturellen Gehirnmutante von Drosophila melanogaster. Diplom thesis, University of Würzburg.

Götz KG (1980) Visual guidance in Drosophila. In: Development and neurobiology of Drosophila (Siddiqi O, Babu P, Hall LM, Hall C, eds), pp 391-407. New York: Plenum.

Graham D (1979) Effects of circum-oesophageal lesion on the behaviour of the stick insect Carausius morosus. II. Changes in walking coordination. Biol Cybern 32:147-152.

Graham D (1985) Pattern and control of walking in insects. Adv Insect Physiol 18:31-140.

Hanesch U (1987) Der Zentralkomplex von Drosophila melanogaster. $\mathrm{PhD}$ thesis, University of Würzburg.

Hanesch U, Fischbach KF, Heisenberg M (1989) Neuronal architecture of the central complex in Drosophila melanogaster. Cell Tissue Res 257:343-366.

Heisenberg M, Böhl K (1979) Isolation of anatomical brain mutants of Drosophila by histological means. Z Naturforsch 34c:143-147.
Heisenberg M, Borst A, Wagner S, Byers D (1985) Drosophila mushroom body mutants are deficient in olfactory learning. J Neurogenet 2:1-30.

Hinton CW (1955) The behavior of an unstable ring chromosome of Drosophila. Genetics 40:951-961.

Homberg $U$ (1987) Structure and functions of the central complex in inscets. In: Arthropod brain: its cvolution, devclopment, structure and functions (Gupta AP, ed), pp 347-367. New York: Wiley.

Huber F (1959) Auslösung von Bewegungsmustern durch elektrische Reizung des Oberschlundganglions bei Orthopteren (Saltatoria, Gryllidae, Acrididae). Verh Zool Ges [Suppl] 23:248-269.

Huber F (1960) Untersuchungen über die Funktion des Zentralnervensystems und insbesondere des Gehirns bei der Fortbewegung und Lauterzeugung der Grillen. Z Vgl Physiol 44:60-132.

Ilius M (1992) Untersuchungen der visuellen Flugsteuerung bei Mutanten des Gens ellipsoid-body-open (ebo) von Drosophila melanogaster. Diplom thesis, University of Würzburg.

Ilius M, Wolf R, Heisenberg M (1992) Visual control of flight orientation involves the central complex. $J$ Neurogenet, in press.

Kankel DR, Hall JC (1976) Fate mapping of nervous system and other internal tissuc in genctic mosaics of Drosophila melanogaster. Dev Biol 48:1-24.

Kien J (1983) The initiation and maintenance of walking in the locust: an alternative to the command concept. Proc R Soc Lond [Biol] 219: 137-174.

Menzel R, Erber J, Masuhr T (1974) Learning and memory in the honeybee. In: Experimental analysis of insect behavior (Browne LB, ed), pp 195-217. Berlin: Springer.

Otto D (1971) Untersuchungen zur zentralnervösen Kontrolle der Lauterzeugung von Grillen. Z Vgl Physiol 74:227-271.

Savvateeva EV, Kamyshev NG (1981) Behavioral effects of temperature sensitive mutations affecting metabolism of cAMP in Drosophila melanogaster. Pharmacol Biochem Behav 14:603-611.

Savvateeva E, Peresleny I, Peresleny A, Tokmacheva E, Medvedeva A, Sharagina L (1992) Bchavioral and biochemical cffects of the agnostic gene complex. J Neurogenet, in press.

Strauß R, Heisenberg M (1990) Coordination of legs during straight walking and turning in Drosophila melanogaster. J Comp Physiol [A] 167:403-412.

Strauß R, Hanesch U, Kinkelin M, Wolf R, Heisenberg M (1992) nobridge of Drosophila melanogaster-portrait of a structural mutant of the central complex. J Neurogenet 8:125-155. 